\title{
Avaliação de İndices Dopplerfluxométricos da Veia Cava Inferior e do Ducto Venoso em Fetos Submetidos à Transfusão Intravascular
}

\author{
Inferior Vena Cava and Ductus Venosus Doppler Ultrasound Index Evaluation \\ of Fetuses Undergoing Intravascular Transfusion
}

Ana Paula Brum Miranda Lopes, Cézar de Alencar de Lima Rezende, Antônio Carlos Vieira Cabral, Henrique Vitor Leite

\begin{abstract}
RESUM0
Objetivo: avaliar os efeitos da transfusão intravascular nos indices dopplerfluxométricos do ducto venoso e da veia cava inferior (SV/CA) e relacionar essas alterações com o nivel de hemoglobina pré-transfusão intravascular.

Métodos: trata-se de estudo transversal prospectivo. Foram realizadas 62 transfusões intravasculares em 27 fetos de gestações complicadas por isoimunização materno-fetal. Os 62 casos foram divididos em dois grupos: (A) fetos com niveis de hemoglobina pré-transfusão $\leq 10 \mathrm{~g} / \mathrm{dL}$, (B) fetos com niveis de hemoglobina pré-transfusão $>10 \mathrm{~g} / \mathrm{dL}$. Os indices $S V / C A$ e CA/SV foram medidos usando Doppler colorido 6 horas antes e 12 horas após a transfusão. Compararam-se os valores dos indices antes e após as transfusões em todos os 62 casos, $e$ após a transfusão em cada grupo. O teste de Wilcoxon foi usado para comparação. Os resultados foram considerados estatisticamente significativos quando $p<0,05$.

Resultados: quando estudamos todo o grupo (62 casos), não encontramos diferença significativa no indice $C A / S V$ antes e após a transfusão $(p=0,775)$. No entanto, o indice $S V / C A$ apresentou aumento significativo após a transfusão $(p=0,004)$. Nos fetos com níveis de hemoglobina prétransfusão $\leq 10 \mathrm{~g} / \mathrm{dL}$ não foi observada diferença significativa nos valores de ambos os indices $S V / C A$ e $C A / S V$, com $p=0,061$ e p=0,345, respectivamente. Após a transfusão intravascular houve aumento significativo do indice $C A / S V$ em fetos com niveis de hemoglobina pré-transfusão $>10 \mathrm{~g} / \mathrm{dL}(\mathrm{p}=0,049)$, mas $\mathrm{o}$ indice $\mathrm{SV} / \mathrm{CA}$ não se alterou nesse grupo $(p=0,086)$.

Conclusões: o estudo por dopplervelocimetria venosa pode ser útil para a compreensão dos ajustes hemodinâmicos fetais após a transfusão intravascular. O aumento no indice SV/CA sem alteração no índice CA/SV após a transfusão em fetos anêmicos pode ser mecanismo compensatório importante em resposta ao aumento do volume intravascular. O aumento do indice $C A / S V$ em fetos com hemoglobina pré-transfusão $<10 \mathrm{~g} / \mathrm{dL}$ sugere um estado de hipervolemia fetal.
\end{abstract}

PALAVRAS-CHAVE: Transfusão intra-útero. Isoimunização materno-fetal. Dopplerfluxometria venosa.

\section{Introdução}

O desenvolvimento da anemia fetal secundária à isoimunização materna é variável e de-

Centro de Medicina Fetal do Hospital da Clínicas da Universidade Federal de Minas Gerais

Correspondência:

Ana Paula Brum Miranda Lopes

R. Wilson Modesto Ribeiro 55 / 404 - Bairro Ipiranga

31160430 - Belo Horizonte - MG

e-mail: apbrum@zaz.com.br pendente de múltiplos fatores, incluindo a concentração, a intensidade de ligação e o tipo dos anticorpos maternos, o grau de passagem transplacentária desses anticorpos e sua afinidade pelo antígeno fetal além da capacidade do feto em estabelecer resposta efetiva à destruição de hemácias. Geralmente, o desenvolvimento da anemia é lento o suficiente para permitir ao feto mobilizar uma série de respostas protetoras, mantendo-se estável mesmo quanto sua hemoglobina 
cai a níveis tão baixos quanto 15 a $20 \%$ dos valores normais ${ }^{1}$.

Os mecanismos compensatórios utilizados pelo feto em resposta à anemia incluem principalmente o estímulo à eritropoese, o aumento do débito cardíaco e a alteração do fluxo sangüíneo regional. Desse modo, o feto com anemia compensada, embora apresente uma queda do volume de oxigênio carreado pelo sangue, mantém sua pressão parcial de $\mathrm{O}_{2}$ e $\mathrm{CO}_{2}$ inalterada ${ }^{2-5}$.

No entanto, apesar de o sistema circulatório fetal possuir reserva considerável que lhe permite adaptar-se a um nivel muito baixo de transporte de $\mathrm{O}_{2}$ em algum momento, na dependência de características individuais, da taxa de queda e do nivel absoluto de hemoglobina, esses mecanismos compensatórios falharão. O feto com anemia descompensada apresentará, então, sinais de hipóxia, acidemia, hidropisia, insuficiência cardiaca e finalmente óbito ${ }^{6,7}$.

A transfusão de sangue intravascular (TIV) por cordocentese representou avanço significativo no tratamento de fetos anêmicos, sendo atualmente a via de escolha, principalmente na abordagem de fetos gravemente acometidos, uma vez que permite correção muito mais eficaz e fisiológica da concentração de hemoglobina, melhorando de forma significativa o prognóstico fetal ${ }^{8}$.

Como o feto anêmico possui um volume intravascular já expandido, a introdução de sangue durante a TIV poderia causar uma sobrecarga de volume, levando a descompensação cardíaca de um feto em seu limite de capacidade de resposta. Porém, o feto anêmico provavelmente lança mão de mecanismos que lhe permitem nova adaptação de sua circulação, de modo que essa expansão aguda de volume seja bem tolerada ${ }^{2,9}$. Experimentos em animais têm demonstrado que após a expansão de volume com sangue total, alguns indicadores hemodinâmicos fetais são modificados, incluindo-se a pressão sangüínea, o débito cardíaco e o fluxo sangüíneo regional ${ }^{2,9}$.

$\mathrm{Na}$ abordagem do feto anêmico, fica clara a importância de se determinar um método, preferencialmente não invasivo, que seja capaz de identificar modificações biofísicas e hemodinâmicas fetais em resposta à correção da anemia e melhora da oxigenação fetal após a TIV. Assim sendo, Manning ${ }^{1}$ relata que a presença de um perfil biofisico fetal normal 24 horas após a TIV é sinal de tratamento bem sucedido e restauração da oxigenação tecidual. Do mesmo modo, Lima ${ }^{10}$, Cabral et al. ${ }^{11}$ e Melo ${ }^{12}$, demonstraram em seus estudos que fetos gravemente anêmicos $(\mathrm{Hb}<7 \mathrm{~g} / \mathrm{dL})$ apresentavam padrão cardiotocográfico não reativo, demonstrando reversão progressiva desse padrão para reativo à medida que as condições fetais eram melhoradas pelo tratamento. Por sua vez, Mari et al. ${ }^{13}$ e Steiner et al. ${ }^{14}$ demonstraram redução no indice de pulsatilidade da artéria cerebral média e diminuição no pico de velocidade média da aorta, respectivamente, em resposta ao aumento do hematócrito fetal. Estudos recentes do fluxo sangüíneo venoso fetal têm demonstrado ser esse compartimento sensivel às alterações hemodinâmicas do feto ${ }^{15}$. No entanto, a avaliação hemodinâmica do feto submetido à TIV por meio da dopplerfluxometria venosa ainda é pouco utilizada, já tendo sido demonstrado que imediatamente após o procedimento ocorre aumento da velocidade no ducto venoso $(\mathrm{DV})^{16}$ e elevação na percentagem de fluxo reverso na veia cava inferior (VCI) ${ }^{17}$.

Com o objetivo de se estudar e compreender as modificações hemodinâmicas promovidas pela TIV na circulação fetal, realizou-se uma avaliação dopplerfluxométrica em fetos submetidos a TIV, visando determinar se após esse procedimento ocorrem alterações nos índices da onda de fluxo na veia cava inferior (CA/SV) e no ducto venoso (SV/CA)

\section{Pacientes e Métodos}

Realizou-se estudo transversal prospectivo, no qual foram acompanhados, no período de março de 2000 a junho de 2001, 27 fetos de gestações únicas sem outras intercorrências maternas ou fetais a não ser a anemia fetal causada por isoimunização materno-fetal, provenientes do Serviço de Pré-natal do Centro de Medicina Fetal-HCUFMG (CEMEFE) e que possuíram indicação para realização de tratamento intra-útero com TIV.

Todas as gestantes foram acompanhadas de acordo com o protocolo de acompanhamento para gestações complicadas por isoimunização maternofetal do HC-UFMG, sendo os dados obtidos armazenados em formulário próprio. As pacientes eram informadas sobre sua doença, possiveis complicações, tipo de tratamento e realização do estudo, assinando o termo de consentimento pós-informado.

De acordo com o protocolo do CEMEFE-HCUFMG foram selecionadas para receber o tratamento intra-uterino as pacientes que apresentavam: idade gestacional inferior a 34 semanas e história de mau passado obstétrico, com grave acometimento perinatal em gestações anteriores causado pela incompatibilidade sangüínea materno-fetal e/ou espectrofotometria do líquido amniótico Ddo 450 nas zonas 2A, 1B e 1A do gráfico de Liley modificado por Robertson ${ }^{18}$ ou, ainda, fetos cujas espectrofotometrias seriadas apresentavam horizontalização ou ascensão da curva, indicando agravamento da hemólise fetal. 
Em todos os 27 fetos foi realizada avaliação dopplerfluxométrica até 6 horas antes e 12 horas após as TIV. Foram obtidas, também, determinações dos níveis de hemoglobina fetal antes e após cada procedimento.

O exame de dopplervelocimetria foi realizado por um único examinador, utilizando o aparelho de Doppler colorido Sonoace 8800 - Medson Co. Ltda., com sonda convexa de $3,5 \mathrm{MHz}$ e filtro acústico de $100 \mathrm{~Hz}$. No momento da realização do exame o examinador desconhecia os valores da hemoglobina fetal. As avaliações foram realizadas durante periodo de inatividade e apnéia fetal.

A VCI foi identificada por meio de corte longitudinal do feto que permite a visualização conjunta do átrio direito, ventrículo direito e aorta, sendo a onda de fluxo obtida no segmento do vaso compreendido entre a veia renal e a veia hepáti$\mathrm{ca}^{19}$.

O DV foi identificado preferencialmente por meio de corte sagital do abdome fetal superior com visualização conjunta da veia umbilical, sendo a obtenção da onda de fluxo realizada próximo à sua origem na veia umbilical ${ }^{20}$.

Após a identificação do vaso, foram obtidos pelo menos três ciclos cardíacos consecutivos e uniformes com onda de fluxo homogêneas. Realizava-se então o traçado manual da onda de fluxo sangüíneo e obtiveram-se os valores de SV e CA de ambos os vasos, calculando-se então os índices estudados: CA/SV na VCI e SV/CA no DV.

As TIV foram realizadas pela mesma equipe, de acordo com o protocolo de CEMEFE-HC-UFMG, sob controle ultra-sonográfico. O sangue foi injetado na veia umbilical, de preferência na inserção placentária do cordão; em caso de dificuldade técnica utilizava-se alça livre. Antes e após as TIV foi obtido $0,5 \mathrm{~mL}$ de sangue para determinação da hemoglobina pré e pós-transfusional por meio de método espectrofotométrico, utilizando aparelho HEMOCUE, previamente calibrado, na própria sala de TIV.

Para a comparação dos resultados obtidos em cada índice (SV/CA e CA/SV) nos períodos pré e pós-tratamento utilizou-se o teste de Wilcoxon. Todos os resultados foram considerados significativos para uma probabilidade de significância inferior a $5 \%(\mathrm{p}<0,05)$.

\section{Resultados}

No período do estudo foram realizadas no Centro de Medicina Fetal CEMEFE-HC-UFMG, 62 transfusões intravasculares em 27 fetos acometidos pela incompatibilidade materno-fetal, re- sultando em média de 2,3 transfusões por paciente.

Após a análise descritiva e comparativa dos índices da VCI e do DV avaliados em todas as 62 TIV, o grupo original (62 casos) foi dividido de acordo com o nivel de hemoglobina fetal obtida por cordocentese, no periodo pré-transfusional, em dois grupos: grupo A (anemia grave) - composto por 31 casos $(50 \%)$, cujo nivel de hemoglobina fetal pré-TIV foi $\leq 10 \mathrm{~g} / \mathrm{dL}$; grupo B - composto de 31 casos cujo nível de hemoglobina fetal pré-TIV foi $>10 \mathrm{~g} / \mathrm{dL}$.

A mediana da idade gestacional no momento da TIV foi de 28 semanas. Os casos acompanhados apresentavam valor mediano de hemoglobina pré-TIV de 10,9 g/dL, indicando anemia leve, sendo que a mediana da hemoglobina pós-TIV foi de $16,0 \mathrm{~g} / \mathrm{dL}$. Nove fetos do total de 27 acompanhados (33\%) apresentavam-se hidrópicos no início do tratamento intra-uterino, todos pertencentes ao grupo A e com $\mathrm{Hb}<7 \mathrm{~g} / \mathrm{dL}$.

Apenas durante dois procedimentos dos 62 realizados $(3,2 \%)$ foi observada a ocorrência de bradicardia fetal, que retornou à freqüência cardiaca fetal normal espontaneamente após a interrupção da TIV. Ambos pertenciam ao grupo B $(\mathrm{Hb}>10 \mathrm{~g} / \mathrm{dL})$. Não houve diferença na freqüência cardiaca fetal antes e após a TIV nos dois grupos estudados: grupo A (fetos com anemia grave) $-144 \pm 7 \mathrm{bpm}$ pré-TIV / $145 \pm 10 \mathrm{bpm}$ pósTIV e, grupo B (fetos com anemia leve/moderada) $-145 \pm 10 \mathrm{bpm}$ pré-TIV / 145 $\pm 10 \mathrm{bpm}$ pós-TIV. Não ocorreram complicações maternas ou obstétricas relacionadas diretamente com o procedimento.

A análise da Tabela 1 demonstra que o índice da VCI (CA/SV) não apresentou alteração quando comparados os valores pré e 12 horas pós-TIV nos 62 casos acompanhados. No entanto, na Tabela 2 observa-se que o índice do DV (SV/CA) elevou-se significativamente no período de 12 horas pós-TIV quando comparado com o valor pré-TIV (Tabela 1).

No grupo de 31 casos com Hb pré-TIV $\leq 10 \mathrm{~g} /$ dL não foi observada alteração significativa nos índices estudados em nenhum dos dois vasos considerados (VCI e DV) quando comparados os valores obtidos no período pré-transfusional e 12 horas após a transfusão (Tabela 2).

Contudo, no grupo dos fetos com anemia leve/moderada ( $\mathrm{Hb}$ pré-TIV $>10 \mathrm{~g} / \mathrm{dL}$ ) pôde-se observar aumento significativo nos valores da relação CA/SV da VCI após a TIV em relação aos valores pré-transfusionais (Tabela 3 ). A relação SV/ CA do DV, por sua vez, não apresentou diferença significativa após a TIV nesse grupo de fetos (Tabela 3$)$. 
Tabela 1 - Valores do índice CA/SV e SVICA no período pré e pós-transfusão intravascular considerando-se todo 0 grupo $(n=62)$

\begin{tabular}{|c|c|c|c|c|c|c|c|c|}
\hline \multirow[b]{2}{*}{ Variável } & \multirow[b]{2}{*}{ Período } & \multicolumn{6}{|c|}{ Medidas Descritivas } & \multirow[b]{2}{*}{$p$} \\
\hline & & $\mathrm{n}$ & Mínimo & Máximo & Mediana & Média & Desvio padrão & \\
\hline CA/SV & Pré & 62 & 0,13 & 1,16 & 0,31 & 0,35 & 0,17 & 0,775 \\
\hline \multirow[t]{2}{*}{ SV/CA } & Pré & 62 & 1,03 & 19.9 & 2,10 & 2,58 & 2.39 & 0,004 \\
\hline & Pós & 62 & 1,40 & 11,5 & 2,42 & 3,28 & 2,13 & Pré < Pós \\
\hline
\end{tabular}

Nota: $\mathrm{O}$ valor $\mathrm{p}$ refere-se ao teste de Wilcoxon

Tabela 2 - Valores dos índices CA/SV e SV/CA no período pré e pós-transfusão intravascular no grupo com Hb pré-transfusão intravascular $\leq 10$ g/dL (grupo A)

\begin{tabular}{lcccccccc}
\hline & & \multicolumn{7}{c}{ Medidas Descritivas } \\
Variável & Período & $\mathbf{n}$ & Mínimo & Máximo & Mediana & Média & Desvio padrão & $\mathbf{p}$ \\
\hline CA/SV & Pré & 31 & 0,13 & 1,16 & 0,36 & 0,41 & 0,22 & 0,345 \\
& Pós & 31 & 0,16 & 1,62 & 0,33 & 0,40 & 0,27 & Pré = Pós \\
SVICA & Pré & 31 & 1,03 & 4,0 & 2,07 & 2,32 & 0,77 & 0,061 \\
& Pós & 31 & 1,40 & 5,90 & 2,43 & 3,02 & 1,34 & Pré = Pós \\
\hline
\end{tabular}

Nota: $O$ valor $p$ refere-se ao teste de Wilcoxon

Tabela 3 - Valores dos índices SV/CA e CA/SV no período pré e pós-transfusão intravascular no grupo com Hb pré-transfusão intravascular >10 g/dL (grupo B).

\begin{tabular}{|c|c|c|c|c|c|c|c|c|}
\hline \multirow[b]{2}{*}{ Variável } & \multirow[b]{2}{*}{ Período } & \multirow[b]{2}{*}{$\mathrm{n}$} & \multirow[b]{2}{*}{ Mínimo } & \multicolumn{3}{|c|}{ Medidas Descritivas } & \multirow[b]{2}{*}{ Desvio padrão } & \multirow[b]{2}{*}{$p$} \\
\hline & & & & Máximo & Mediana & Média & & \\
\hline SV/CA & Pré & 31 & 1,51 & 19,90 & 2,28 & 2,84 & 3,30 & 0,086 \\
\hline \multirow[t]{2}{*}{ CA/SV } & Pré & 31 & 0,20 & 0,43 & 0,29 & 0,29 & 0.06 & 0,049 \\
\hline & Pós & 31 & 0,13 & 0,54 & 0,32 & 0,33 & 0,09 & Pré < Pós \\
\hline
\end{tabular}

Nota: 0 valor $p$ refere-se ao teste de Wilcoxon

\section{Discussão}

A TVI impõe alterações agudas no sistema cardiovascular fetal pelo aumento de volume e viscosidade sangüinea. A literatura tem demonstrado que após esse procedimento observa-se aumento na pré e na pós-carga ${ }^{2,21}$, sendo que essas alterações podem levar ao desenvolvimento de respostas compensatórias pelo feto, as quais podem ser identificadas pela avaliação dopplerfluxométrica.

O estudo do fluxo sangüíneo venoso fetal tem demonstrado ser esse compartimento sensivel às alterações hemodinâmicas do feto. $\mathrm{Na}$ avaliação dopplerfluxométrica venosa fetal dois vasos merecem destaque: o ducto venoso, que é considerado um regulador da circulação fetal, sendo responsável pelo aporte de sangue bem oxigenado para o coração e o cérebro e a veia cava inferior que reflete as alterações hemodinâmicas intracardíacas.

$\mathrm{Na}$ avaliação dopplerfluxométrica desses vasos dois índices de avaliação das ondas de fluxo são mais utilizados na literatura. $\mathrm{O}$ índice CA/SV na VCI usado na avaliação da função cardiaca. O indice SV/CA do DV que expressa a pré-carga do ventrículo direito, refletindo o gradiente de pressão entre o átrio e o ventrículo. Esses dois índices possuem como característica principal o fato de serem ângulo-independentes, refletindo alterações na forma da onda, representando de maneira mais segura as alterações circulatórias.

Nesse estudo observou-se que 12 horas após a correção da anemia ocorreu elevação significativa do índice SV/CA (DV) quando comparado com seus valores pré-transfusão, fato também encontrado por Oepkes et al. ${ }^{16}$ que demonstraram em seu estudo do comportamento dopplerfluxométrico do DV em fetos anêmicos elevação desse índice 24 horas após a correção da anemia e atribuíram esse achado a mecanismo cardiovascular compensatório, desenvolvido pelo feto em resposta ao aumento de volume imposto pela TIV. 
De fato, observou-se também, nesse estudo, que os fetos com correção da anemia não apresentaram sinais de sobrecarga circulatória 12 horas após a TIV, evidenciada pela não-alteração dos valores do índice CA/SC (VCI) após a transfusão. O estudo de Rizzo et al. ${ }^{17}$ demonstra que imediatamente após a TIV (até 2 horas) ocorre uma elevação do índice $\mathrm{CA} / \mathrm{SV}$, com retorno a valores normais $24 \mathrm{hs}$ após o procedimento. Isso sugere que logo após a TIV ocorre uma sobrecarga circulatória, porém o feto é capaz de promover uma readaptação hemodinâmica que o permite estabilizar sua circulação.

Hecher et al. ${ }^{22}$ e Rizzo et al. ${ }^{17}$ demonstraram em seus respectivos estudos que fetos com hipóxia acentuada e acidemia apresentam elevação dos índices SV/CA e CA/SV atribuída ao comprometimento da contração cardíaca pela hipóxia miocárdica e conseqüente descompensação cardiaca fetal. Nesse estudo, observou-se também que fetos muito acometidos pela anemia ( $\mathrm{HB}<10 \mathrm{~g} / \mathrm{dL}$ ) não apresentavam alterações significativas nos valores dos índices estudados após a TIV, sugerindo comprometimento importante da função cardíaca nesses fetos decorrente da anemia grave, dificultando o desenvolvimento de resposta compensatória após a transfusão.

Os fetos com anemia leve ( $\mathrm{Hb}>10 \mathrm{~g} / \mathrm{dL})$ apresentaram uma elevação significativa do índice CA/ SV 12 horas após a TIV. De acordo com Okamura et al. $^{23}$, o aumento nesse índice da VCI, deve alertar para a possibilidade da presença de insuficiência cardiaca. Como o feto possui capacidade limitada de aumentar sua contractilidade miocárdica em resposta ao aumento de volume ${ }^{24}$, o aumento observado no índice CA/SV nos fetos com anemia leve sugere sobrecarga circulatória conseqüente a um aumento do volume sangüineo, sem elevação correspondente em sua contração miocárdica.

A avaliação dopplerfluxométrica da circulação venosa 12 horas após a TIV auxilia na compreensão da resposta hemodinâmica fetal após a TIV, sendo a elevação do índice SV/CA sugestiva de rearranjos circulatórios compensatórios desenvolvidos pelo feto após a correção da anemia e a elevação do índice CA/SV indicativa de sobrecarga de volume.

\section{ABSTRACT}

Purpose: to evaluate the effect of intravascular transfusion on ductus venosus and inferior vena cava Doppler ultrasound indexes $(S V / C A)$ and to relate it to hemoglobin levels before transfusion.
Methods: this is a transversal prospective study. A total of 62 intravascular transfusions were performed in 27 fetuses from pregnancies with red blood cell isoimmunization. The 62 cases were divided into two groups: (1) fetuses with hemoglobin levels before transfusion $\leq 10 \mathrm{~g} / \mathrm{dL}$ and (2) fetuses with hemoglobin levels before transfusion $>10 \mathrm{~g} / \mathrm{dL}$. The SV/ $C A$ and $C A / S V$ indexes were measured using color Doppler ultrasound $6 \mathrm{~h}$ before and $12 \mathrm{~h}$ after intravascular transfusion. The index values before and after transfusion in all 62 cases were compared. Thereafter we compared these indexes before and after transfusion regarding each group. The Wilcoxon test was used and the results were considered statiscally significant when $p<0.05$.

Results: when we studied the whole group (62 cases) no significant difference was observed between the CA/SV index before and after transfusion $(p=0.775)$. On the other hand, a significant increase in the SV/CA index was observed after transfusion $(p=0.004)$. No significant differences were observed in both the SV/CA and CA/SV indexes before and after transfusion in the group of fetuses with hemoglobin levels before transfusion $\leq 10 \mathrm{~g} / \mathrm{dL}(p=0.061$ and $p=0.345$, respectively). There was a significant increase in the CA/SV index after transfusion in fetuses with hemoglobin levels before transfusion $>10 \mathrm{~g} / \mathrm{dL}(p=0.049)$, but the SV/CA index did not change in this group $(p=0.086)$.

Conclusion: venous Doppler study may be useful to understand fetal hemodynamic adjustment after intravascular transfusion. An increase in SV/CA without change in CA/SV after transfusion in anemic fetuses may be an important compensatory mechanism to increase intravascular volume. The increase in CA/SV index in fetuses with hemoglobin levels before transfusion $<10 \mathrm{~g} /$ dL suggests a state of fetal hypervolemia.

KEY WORDS: Intrauterine blood transfusion. Maternal-fetal isoimmunization, venous. Doppler ultrasound.

\section{Referências}

1. Manning FA. Gravidez aloimune: diagnóstico e conduta. In: Manning FA, editor. Medicina Fetal: perfil biofísico, princípios e aplicabilidade clínica. $1^{\text {a }}$ ed. Rio de Janeiro: Revinter; 2000. p.391-445.

2. Fan FC, Chen RY, Schuessler GB, Chien S. Effects of hematocrit variations on regional hemodynamics and oxygen transport in the dog. Am J Physiol 1980; 238:H545-22.

3. Copel JA, Grannum PA, Green JJ, et al. Fetal cardiac output in the isoimmunized pregnancy: a pulsed Doppler-echocardiographic study of patients undergoing intravascular intrauterine transfusion. Am J Obstet Gynecol 1989; 161:361-5.

4. Rizzo G, Nicolaides KH, Arduini D, Campbell S Effects of intravascular fetal blood transfusion on fetal intracardiac doppler velocity waveforms. Am J Obstet Gynecol 1990; 163:1231-8. 
5. Bowman JM. Hemolytic disease. In: Creasy RK, Resnik R, editors. Maternal-fetal medicine. $4^{\text {th }}$ ed. Philadelphia: Saunders; 1999. p.736-7.

6. Soothill PW, Nicolaides $\mathrm{KH}$, Rodeck CH, Clewell WH, Lindridge J. Relationship of fetal hemoglobin and oxygen content to lactate concentration in $\mathrm{Rh}$ isoimmunized pregnancies. Obstet Gynecol 1987; 69:268-71.

7. Nicolaides $\mathrm{KH}$, Rodeck $\mathrm{CH}$, Millar DS, Misbahan RS. Fetal haematology in rhesus isoimmunization. $\mathrm{Br}$ Med J (Clin Res Ed) 1985; 290:661-3.

8. Cabral ACV, Taveira MR, Lopes APBM, Pereira AK, Leite HV. Transfusão intra-uterina na isoimunização materna pelo fator Rh. Rev Bras Ginecol Obstet 2001; 23:299-303.

9. Fumia FD, Edelstone DI, Holzman IR. Blood flow and oxygen delivery to fetal organs as functions of fetal hematocrit. Am J Obstet Gynecol 1984; 150:274-82.

10.Lima MAM. Carditocografia no acompanhamento de gestações complicadas pela isoimunização materna pelo fator Rh [dissertação]. Belo Horizonte: Universidade Federal de Minas Gerais; 1986.

11.Cabral ACV, Melo VH, Silva DR, Rezende CAL, Corrêa MD. Cardiotocografia no acompanhamento de fetos submetidos à transfusão intravascular. J Bras Ginecol 1990; 100:367-70.

12. Melo IG. Cardiotocografia anteparto em fetos de gestantes isoimunizadas [dissertação]. Belo Horizonte: Universidade Federal de Minas Gerais; 2000.

13.Mari G, Moise KJ Jr, Deter RL, Carpenter RJ Jr. Flow velocity waveforms of the umbilical and cerebral arteries before and after intravascular transfusion Obstet Gynecol 1990; 75:584-9.

14. Steiner H, Schaffer H, Spitzer D, Batka M, Graf AH, Staudach A. The relationship between peak velocity in the fetal descending aorta and hematocrit in rhesus isoimmunization. Obstet Gynecol 1995; 85:659-62.

15.Hecher K, Campbell S, Snijders R, Nicolaides K. Reference ranges for fetal venous and atrioventricular blood flow parameters ultrasound. Ultrasound Obstet Gynecol 1994; 4:381-90.
16. Oepkes D, Vandenbussche FP, Van Bel F, Kanhai HH. Fetal ductus venosus blood flow velocities before and after transfusion in red-cell alloimmunized pregnancies. Obstet Gynecol 1993; 82:237-41.

17.Rizzo G, Arduini D, Romanini C. Fetal cardiac function in anemia. In: Arduini D, Rizzo G, Romanini C, editors. Fetal Cardiac Function. $1^{\text {st }}$ ed. New York: Parthenon; 1995. p.103-8.

18. Robertson JG. Examination of amniotic fluid in rhesus isoimunization. BMJ 1964; 2:147-52.

19.Rizzo G, Arduini D, Romanini C. Fetal Doppler echoardiography: principles, techniques and reference limits. In: Arduini D, Rizzo G, Romaini C. Fetal cardiac function. New York: Parthenon, 1995; 3: 33-9.

20.Kiserud T, Eik-Nes SH. The fetal ductus venosus In: Copel JA, Reed KL, editors. Doppler Ultrasound in Obstetric and Gynecology. $1^{\text {st }}$ ed. New York: Raven Press; 1995. p.297-305.

21.Moise KJ Jr, Mari G, Fisher DJ, Huhta JC, Cano LE, Carpenter RJ Jr. Acute fetal hemodynamic alterations after intrauterine transfusion for treatment of severe red blood cell alloimmunization. Am J Obstet Gynecol 1990; 163:776-84.

22.Hecher K, Snijders R, Campbell S, Nicolaides K. Fetal venous, intracardiac, and arterial blood flow measurements in intrauterine growth retardation: relationship with fetal blood gases. Circulation 1995; 173:10-5.

23. Okamura K, Murotsuki J, Kobayaski M, et al. Umbilical and venous pressure and Doppler flow pattern of inferior vena cava in the fetus. Am J Perinatol 1994; 11:255-9.

24.Walker AM. Physiological control of fetal cardiovascular system. In: Harding $\mathrm{H}$, editor. The Fetal Cardiovascular System. $1^{\text {st }}$ ed. Philadelphia: Saunders; 1982. p.287-310.

Recebido em: 30/1/2002 Aceito com modificações em: 9/12/2002

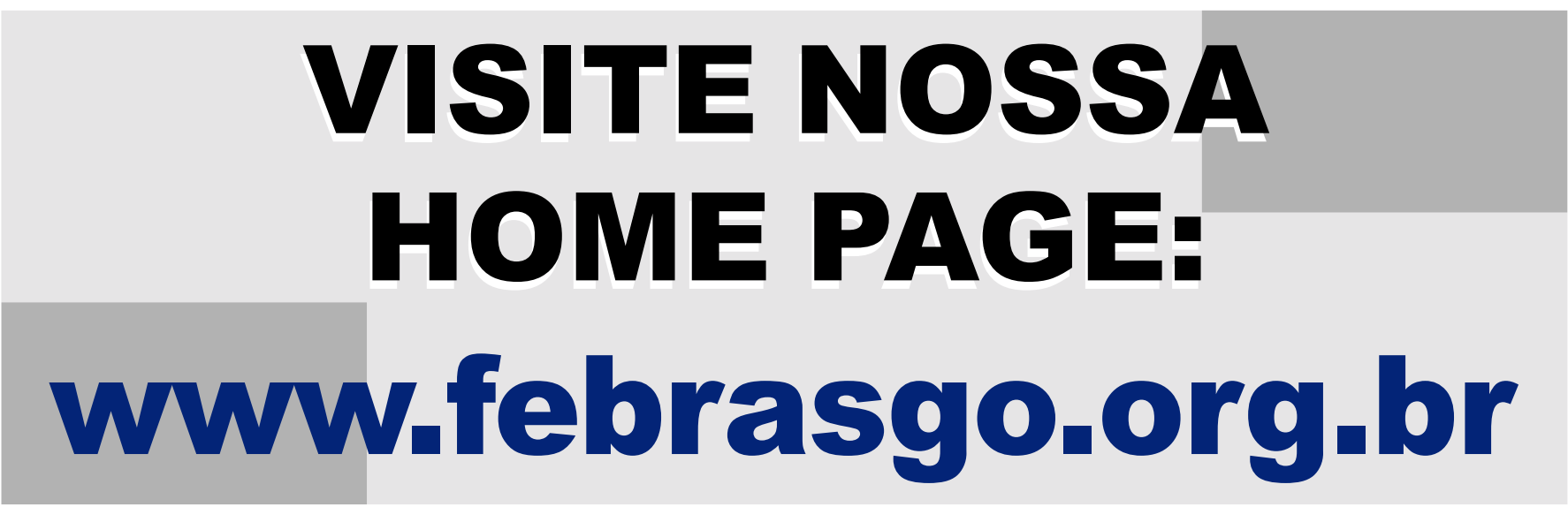

